\title{
ON HYPERSPACES OF POLYHEDRA
}

\author{
KATSURO SAKAI
}

(Communicated by James E. West)

\begin{abstract}
Let $Q=[-1,1]^{\omega}$ be the Hilbert cube and
$Q_{f}=\left\{\left(x_{i}\right) \in Q \mid x_{i}=0\right.$ except for finitely many $\left.i\right\}$.
\end{abstract}

For a compact connected polyhedron $X$ with $\operatorname{dim} X>0$, the hyperspaces of (nonempty) subcompacta, subcontinua, and compact subpolyhedra of $X$ are denoted by $2^{X}, C(X)$, and $\operatorname{Pol}(X)$, respectively. And let $C^{\mathrm{Pol}}(X)=C(X) \cap$ $\operatorname{Pol}(X)$. It is shown that the pair $\left(2^{X}, \operatorname{Pol}(X)\right)$ is homeomorphic to $\left(Q, Q_{f}\right)$. In case $X$ has no free arc, it is also proved that the pair $\left(C(X), C^{\mathrm{Pol}}(X)\right)$ is homeomorphic to $\left(Q, Q_{f}\right)$.

\section{INTRODUCTION}

The hyperspace $2^{X}$ of nonempty subcompacta of a nondegenerate Peano

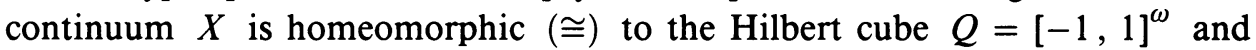
if $X$ has no free arc then the hyperspace $C(X)$ of nonempty subcontinua of $X$ is also homeomorphic to $Q$ [CS ${ }_{1}$ ] (cf. [To]). All hyperspaces in this paper are topologized by the Hausdorff metric. The subset $s=(-1,1)^{\omega}$ of $Q$ is called the pseudo-interior of $Q$ and $Q \backslash s$ the pseudo-boundary of $Q$. Let $\Sigma=\left\{\left(x_{i}\right) \in s|\sup | x_{i} \mid<1\right\}$. Then $(Q, \Sigma) \cong(Q, Q \backslash s)$ and these sets $\Sigma$ and $Q \backslash s$ are topologically characterized as cap sets for $Q$ [An]. The hyperspace $2^{X}$ has several cap sets which naturally appear as hyperspaces $\left[\mathrm{Mi}_{1}\right]\left(\mathrm{cf} .\left[\mathrm{Mi}_{2}\right]\right.$ and $[\mathrm{CM}])$. Let us consider the following subsets of $Q$ :

$$
\sigma=\left\{\left(x_{i}\right) \in s \mid x_{i}=0 \text { except for finitely many } i\right\}
$$

and

$$
Q_{f}=\left\{\left(x_{i}\right) \in Q \mid x_{i}=0 \text { except for finitely many } i\right\} .
$$

Then $(Q, \sigma) \cong\left(Q, Q_{f}\right)$ and these sets $\sigma$ and $Q_{f}$ are topologically characterized as fd-cap sets for $Q$ [An]. If $X$ is a countable union of finite-dimensional (abbreviated fd) compacta, the hyperspace $\mathscr{F}(X)$ of nonempty finite sets in

Received by the editors March 22, 1989 and, in revised form, September 16, 1989; presented at the General Topology Symposium, Kagawa University, December 12, 1989 and to the Mathematical Society of Japan, April 2, 1990.

1980 Mathematics Subject Classification (1985 Revision). Primary 54B20, 51M20, 57N20.

Key words and phrases. Hyperspaces, polyhedron, Hilbert cube, fd-cap set. 
$X$ is an fd-cap set for $2^{X}$, that is, $\left(2^{X}, \mathscr{F}(X)\right) \cong(Q, \sigma)$ [CN]. It seems that a "natural" fd-cap set (or cap set) for $C(X)$ is unknown. The purpose of this paper is to obtain a natural fd-cap set for $C(X)$ in case $X$ is a polyhedron. For fd-cap (or cap) sets, we refer the reader to [Ch].

For a compact connected polyhedron $X$, the hyperspace of nonempty compact subpolyhedra of $X$ is denoted by $\operatorname{Pol}(X)$. And let $C^{\mathrm{Pol}}(X)=C(X) \cap$ $\operatorname{Pol}(X)$, the hyperspace of nonempty compact connected subpolyhedra of $X$. The following theorem is our main result.

Main Theorem. Let $X$ be a compact connected polyhedron with $\operatorname{dim} X>0$. Then $\operatorname{Pol}(X)$ is an fd-cap set for $2^{X}$, that is, $\left(2^{X}, \operatorname{Pol}(X)\right) \cong(Q, \sigma)$. In case $X$ has no free arcs, $C^{\mathrm{Pol}}(X)$ is an fd-cap set for $C(X)$, that is, $\left(C(X), C^{\mathrm{Pol}}(X)\right)$ $\cong(Q, \sigma)$.

\section{Preliminaries}

Let $M=(M, d)$ be a metric space. For $x \in M$ and $A \subset M$, we denote $d(x, A)=\inf \{d(x, a) \mid a \in A\}$. The diameter of $A \subset M$ is denoted by $\operatorname{diam} A$. For a collection $\mathscr{A}$ of subsets of $M$, we denote mesh $\mathscr{A}=$ $\sup \{\operatorname{diam} A \mid A \in \mathscr{A}\}$. For maps $f, g: Y \rightarrow M$ from a space $Y$ to $M$, let $d(f, g)=\sup \{d(f(x), g(x)) \mid x \in Y\}$. A closed set $A$ in $M$ is called a $Z$-set if for each map $f: Q \rightarrow M$ and $\varepsilon>0$, there is a map $g: Q \rightarrow M \backslash A$ with $d(f, g)<\varepsilon$. A countable union of $Z$-sets in $M$ is called a $Z_{\sigma}$-set. An fd-cap set (or a cap set) for $M$ is a set $N=\bigcup_{i \in \mathbf{N}} N_{i} \subset M$, where $N_{1} \subset N_{2} \subset \ldots$ are fd compact $Z$-sets (or compact $Z$-sets) in $M$ and the following condition is satisfied:

For each fd compactum (or compactum) $A \subset M, i \in N$ and $\varepsilon>0$, there is an embedding $h: A \rightarrow N_{j}$ of $A$ into some $N_{j}\left(\supset N_{i}\right)$ such that $h \mid A \cap N_{i}=$ id and $d(h, \mathrm{id})<\varepsilon$.

The following is due to R. D. Anderson [An] (cf. [Ch]).

1.1. Lemma. In order that $(M, N) \cong(Q, \sigma) \quad($ or $(M, N) \cong(Q, \Sigma))$, it is necessary and sufficient that $M \cong Q$ and $N$ is an fd-cap set (or a cap set) for $M$.

Throughout the paper, let $X=|K|$ denote a compact connected polyhedron with $\operatorname{dim} X>0$ and $K$ a triangulation. We consider $X$ as a (rectilinear) subpolyhedron of some $\mathbf{R}^{n}$. By identifying $x \in X$ and $\{x\} \in 2^{X}$, we consider $X \subset 2^{X}$. Do not confuse $X \in 2^{X}$ with $X \subset 2^{X}$. Let $\rho$ denote the path length metric on $X$ defined by the metric inherited from Euclidean metric on $\mathbf{R}^{n}$ (cf. [CN, Lemma 4.3]). Then $\rho$ is convex in the sense of Menger [Me] and Euclidean on each simplex of $K$. By $\rho_{H}$, we denote the Hausdorff metric on $2^{X}$ defined by

$$
\rho_{H}(A, B)=\max \{\sup \{\rho(x, A) \mid x \in B\}, \sup \{\rho(x, B) \mid x \in A\}\} .
$$


The barycenter, interior, and boundary of $\tau \in K$ are denoted by $\hat{\tau}$, $\dot{\tau}$, and $\partial \tau$ and the star and link of $\tau \in K$ are denoted by $\operatorname{St}(\tau, K)$ and $\operatorname{Lk}(\tau, K)$, respectively. For each $v \in K^{0}$, let $\mathrm{O}(v, K)=|\operatorname{St}(v, K)| \backslash|\mathrm{Lk}(v, K)|$. Then $\left\{\mathrm{O}(v, K) \mid v \in K^{0}\right\}$ is an open cover of $X$. The $n$-skeleton and the $n$th barycentric subdivision of $K$ are denoted by $K^{n}$ and $\operatorname{Sd}^{n} K$, respectively. We write $\mathrm{Sd}^{1} K=\mathrm{Sd} K$.

\section{THE HYPERSPACE $\operatorname{Pol}(X)$}

In this section, we prove the first half of the Main Theorem, that is, $\left(2^{X}\right.$, $\operatorname{Pol}(X)) \cong(Q, \sigma)$. For each $n \in \mathbf{N}$, let $C_{n}(X)$ denote the subspace of $2^{X}$ consisting of all compacta with at most $n$ components. Then each $C_{n}(X)$ is a $Z$-set in $2^{X}$. In fact, for each $0<\varepsilon<\operatorname{diam} X$, we define $\kappa: 2^{X} \rightarrow 2^{X}$ by $\kappa(A)=\{x \in X \mid \rho(x, A) \leq \varepsilon\}$. Then $\kappa$ is continuous [Na, Corollary 3.4]. Since $\mathscr{F}(X)$ is an fd-cap set for $2^{X}$, there is a map $\varphi: 2^{X} \rightarrow \mathscr{F}(X)$ with $d(\varphi$, id $)<\varepsilon / n$. As easily observed, each $\varphi \kappa(A)$ has at least $n+1$ components. Thus we have a map $\varphi \kappa: 2^{X} \rightarrow 2^{X} \backslash C_{n}(X)$ with $d(\varphi \kappa$, id $)<(n+1) \cdot \varepsilon / n$. Now let $C_{\omega}(X)=\bigcup_{n \in \mathrm{N}} C_{n}(X)$ be the subset of $2^{X}$ consisting of all compacta with finitely many components. Then $C_{\omega}(X)$ is a $Z_{\sigma}$-set in $2^{X}$ and $\mathscr{F}(X) \subset$ $\operatorname{Pol}(X) \subset C_{\omega}(X)$. The first half of the theorem follows from [Ch, Lemma 4.2] and the following lemma.

2.1. Lemma. $\operatorname{Pol}(X)$ is $\sigma$-fd-compact, that is, a countable union of fd compacta.

Proof. Let $Y$ be the convex hull of $X$ in $\mathbf{R}^{n}$. Then $\operatorname{Pol}(X)$ is closed in $\operatorname{Pol}(Y)$. If $\operatorname{Pol}(Y)$ is $\sigma$-fd-compact, so is $\operatorname{Pol}(X)$. Thus we may assume that $X$ is a compact convex set in $\mathbf{R}^{n}$. A triangulation of $P \in \operatorname{Pol}(X)$ is said to be minimal if the number of vertices is minimal among all triangulation of $P$. Let $\mathscr{F}$ be the set of all finite subcomplexes of the countable infinite full complex $\Delta^{\infty}$. For each $L \in \mathscr{F}$, let $\operatorname{Pol}_{L}(X)$ be the subset of $\operatorname{Pol}(X)$ consisting of all polyhedra with a minimal triangulation which is simplicially isomorphic to $L$. It is straightforward to show that $\operatorname{Pol}_{L}(X)$ is locally homeomorphic to $F_{n}(X) \backslash F_{n-1}(X)$, where $n$ is a number of vertices of $L$ and $F_{n}(X)=\{A \in$ $2^{X} \mid$ card $\left.A \leq n\right\}$. Since $F_{n}(X) \backslash F_{n-1}(X)$ is $\sigma$-fd-compact (cf. the proof of [CN, Lemma 3.1]), so is $\operatorname{Pol}_{L}(X)$. Therefore $\operatorname{Pol}(X)=\bigcup_{L \in \mathscr{F}} \operatorname{Pol}_{L}(X)$ is also $\sigma$-fd-compact since $\mathscr{F}$ is countable.

\section{THE HYPERSPACE $C^{\mathrm{Pol}}(X)$}

In this section, we prove the second half of the Main Theorem, that is, $\left(C(X), C^{\mathrm{Pol}}(X)\right) \cong(Q, \sigma)$ if $X$ has no free arc. First we prove the following lemma in which $X$ may have a free arc.

3.1. Lemma. For each $\varepsilon>0$ there exists a $\delta>0$ such that each $\mathscr{A} \subset \operatorname{Pol}(X)$ with $\operatorname{diam} \mathscr{A}<\delta$ is contractible in a set $\mathscr{B} \subset \operatorname{Pol}(X)$ such that $\operatorname{diam} \mathscr{B}<\varepsilon$, 
each $B \in \mathscr{B}$ contains some $A \in \mathscr{A}$ and each component of $B$ meets $A$, hence $\mathscr{B} \subset C^{\mathrm{Pol}}(X)$ if $\mathscr{A} \subset C^{\mathrm{Pol}}(X)$.

Proof. Subdividing $K$, we may assume that mesh $K<\varepsilon / 4$. Choose $\delta>0$ so that the closed $\delta$-neighborhood of each $|\operatorname{St}(v, \operatorname{Sd} K)|$ in $|K|$ is contained in $O(v, K)$. For each $\mathscr{A} \subset \operatorname{Pol}(X)$ and $\operatorname{diam} \mathscr{A}<\delta$, take an $A_{0} \in \mathscr{A}$ and let $\left\{v_{1}, \ldots, v_{n}\right\}=\left\{v \in K^{0}\left|A_{0} \cap\right| \operatorname{St}(v, \operatorname{Sd} K) \mid \neq \varnothing\right\}$ and $V_{i}$ be the $\delta$ neighborhood of $\left|\operatorname{St}\left(v_{i}, \operatorname{Sd} K\right)\right|$ in $|K|$. Then it follows that each $A \in \mathscr{A}$ is contained in $\bigcup_{i=1}^{n} V_{i}$ and meets each $V_{i}$ because for each $a \in A$ there is an $a_{0} \in A_{0}$ with $\rho\left(a, a_{0}\right)<\delta$ and conversely for each $a_{0} \in A_{0}$ there is an $a \in A$ with $\rho\left(a, a_{0}\right)<\delta$.

We observe that if $A \subset \bigcup_{i=1}^{n}\left|\operatorname{St}\left(v_{i}, K\right)\right|$ and $A$ meets each $\left|\operatorname{St}\left(v_{i}, K\right)\right|$, then $\rho_{H}\left(A, A_{0}\right)<\varepsilon / 2$. In fact, each $a \in A$ is contained in some $\left|\operatorname{St}\left(v_{i}, K\right)\right|$. Then $\rho\left(a, v_{i}\right) \leq \operatorname{mesh} K<\varepsilon / 4$. Since $A_{0} \cap\left|\operatorname{St}\left(v_{i}, \operatorname{Sd} K\right)\right| \neq \varnothing$, we have an $a_{0} \in A_{0}$ such that $\rho\left(a_{0}, v_{i}\right)<\varepsilon / 4$, hence $\rho\left(a, a_{0}\right)<\varepsilon / 2$. Conversely, each $a_{0} \in A_{0}$ is contained in some $\left|\operatorname{St}\left(v_{i}, \operatorname{Sd} K\right)\right|$. Then $\rho\left(a_{0}, v_{i}\right)<\varepsilon / 4$. Since $A \cap\left|\operatorname{St}\left(v_{i}, K\right)\right| \neq \varnothing$, we have an $a \in A$ such that $\rho\left(a, v_{i}\right)<\varepsilon / 4$; hence $\rho\left(a, a_{0}\right)<\varepsilon / 2$. Therefore $\rho_{H}\left(A, A_{0}\right)<\varepsilon / 2$.

Now we define a homotopy $\varphi: \mathscr{A} \times I \rightarrow \operatorname{Pol}(X)$ as follows:

$$
\varphi(A, t)=\bigcup_{i=1}^{n}\left\{s k_{i}(a) \cdot v_{i}+(1-s) k_{i}(a) \cdot a|a \in A \cap| \operatorname{St}\left(v_{i}, K\right) \mid, s \in[0, t]\right\} .
$$

where $k_{i}:\left|\operatorname{St}\left(v_{i}, K\right)\right| \rightarrow I$ is a map with $k_{i}\left(\operatorname{cl} V_{i}\right)=1$ and $k_{i}\left(\left|\operatorname{Lk}\left(v_{i}, K\right)\right|\right)=0$. Then $\varphi_{0}=$ id and $A \cup\left\{v_{1}, \ldots, v_{n}\right\} \subset \varphi_{1}(A)$ for each $A \in \mathscr{A}$. By the fact observed above, $\rho_{H}\left(\varphi_{t}(A), A_{0}\right)<\varepsilon / 2$ for each $(A, t) \in \mathscr{A} \times I$. And we define a homotopy $\psi: \mathscr{A} \times I \rightarrow \operatorname{Pol}(X)$ as follows:

$$
\psi(A, t)=\varphi_{1}(A) \cup \bigcup_{i=1}^{n}\left\{(1-s) \cdot v_{i}+s \cdot x|x \in| \operatorname{St}\left(v_{i}, K\right) \mid, s \in[0, t]\right\} .
$$

Then $\psi_{0}=\varphi_{1}$ and $\psi_{1}(A)=\bigcup_{i=1}^{n}\left|\operatorname{St}\left(v_{i}, K\right)\right|$ for each $A \in \mathscr{A}$. Again by the fact observed above, $\rho_{H}\left(\psi_{t}(A), A_{0}\right)<\varepsilon / 2$ for each $(A, t) \in \mathscr{A} \times I$. Then $\mathscr{A}$ is contractible in the set $\mathscr{B}=\varphi(\mathscr{A} \times I) \cup \psi(\mathscr{A} \times I) \subset \operatorname{Pol}(X)$ with diam $\mathscr{B}<\varepsilon$. Observe that each $B \in \mathscr{B}$ contains some $A \in \mathscr{A}$ and each component of $B$ meets $A$.

Hereafter we assume that $X$ has no free arc.

3.2. Lemma. $C^{\mathrm{Pol}}(X)$ is a $Z_{\sigma}$-set in $C(X)$.

Proof. By Lemma 2.1, $C^{\mathrm{Pol}}(X)$ is $\sigma$-compact, so $F_{\sigma}$ in $C(X)$. Then it suffices to show that for each $\varepsilon>0$, there is a map $\varphi: C(X) \rightarrow C(X) \backslash \operatorname{Pol}(X)$ with $\rho_{H}(\varphi$, id $)<\varepsilon$. Subdividing $K$, we may assume that mesh $K<\varepsilon / 2$. Let $\left\{\tau_{i} \mid i=1, \ldots, n\right\}$ be the set of principal (= maximal dimensional) simplexes of $K$. Then $X=\bigcup_{i=1}^{n} \tau_{i}$. Choose Euclidean balls $B_{i}$ in $\dot{\tau}_{i}=$ int $\tau_{i}$ such that $\operatorname{dim} B_{i}=\operatorname{dim} \tau_{i} \geq 2$ and set $Y=X \backslash \bigcup_{i=1}^{n} \dot{B}_{i}$. Since $C\left(\partial B_{i}\right)$ is an AR, we 
have a map $f_{i}: B_{i} \rightarrow C\left(\partial B_{i}\right)$ such that $f_{i} \mid \partial B_{i}=$ id. Let $f: X \rightarrow C(Y)$ be the map defined by $f \mid Y=$ id and $f \mid B_{i}=f_{i}$. Then $f$ induces the map $\theta: C(X) \rightarrow C(Y) \subset C(X)$ defined by $\theta(A)=\bigcup\{f(a) \mid a \in A\}$ (cf. [Ke]). It is easy to see that $\rho_{H}(\theta$, id $)<\varepsilon / 2$. If $A$ contains some $B_{i}$, then $\theta(A)$ contains $\partial B_{i}$ but misses $\dot{B}_{i}$ whence any point of $\partial B_{i}$ has no cone neighborhood in $\theta(A)$ (because $\operatorname{dim} B_{i} \geq 2$ ), that is, $\theta(A)$ is not a polyhedron (cf. [RS]). Let $\kappa: C(X) \rightarrow C(X)$ be the map defined by $\kappa(A)=\{x \in X \mid \rho(x, A) \leq \varepsilon / 2\}$. Clearly $\rho_{H}(\kappa$, id $) \leq \varepsilon / 2$ and each $\kappa(A)$ contains some $B_{i}$. Thus we have the map $\varphi=\theta \circ \kappa: C(X) \rightarrow C(X) \backslash \operatorname{Pol}(X)$ with $\rho_{H}(\varphi$, id $)<\varepsilon$.

For each $n \in \mathbf{N}$, let $Y_{n}=\left|\left(\operatorname{Sd}^{2 n} K\right)^{1}\right|$ and

$$
P_{n}=\left\{A \in C\left(Y_{n}\right) \mid \exists \tau \in \mathrm{Sd}^{2 n} K \text { s.t. } \operatorname{dim} \tau=1 \text { and } \tau \subset A\right\} .
$$

Then each $P_{n}$ is closed in $C\left(Y_{n}\right)$, and is therefore fd compact.

3.3. Lemma. For each $n \in \mathbf{N}$, there exists an embedding $\xi: P_{n} \times I \rightarrow P_{n+1}$.

Proof. We use the path length metric $\rho^{\prime}$ on $Y_{n+1}$. One should note that $\rho^{\prime}$ is not the restriction of $\rho$. Let

$$
\begin{aligned}
\beta & =\min \left\{\rho^{\prime}\left(v, v^{\prime}\right) \mid v \neq v^{\prime} \in\left(\operatorname{Sd}^{2 n+2} K\right)^{0}\right\} \\
& \leq \frac{1}{4} \cdot \min \left\{\rho^{\prime}\left(v, v^{\prime}\right) \mid v \neq v^{\prime} \in\left(\operatorname{Sd}^{2 n} K\right)^{0}\right\} .
\end{aligned}
$$

Then for points $x$ and $x^{\prime}$ in any connected subgraph $Z$ of $Y_{n+1}$, if $\rho^{\prime}\left(x, x^{\prime}\right)<$ $\beta$ then $\rho^{\prime}\left(x, x^{\prime}\right)$ is the distance between $x$ and $x^{\prime}$ with respect to the path length metric on $Z$. Let $\kappa: P_{n} \times I \rightarrow P_{n}$ be the map defined by $\kappa(A, t)=\{x \in$ $\left.Y_{n} \mid \rho^{\prime}(x, A) \leq t \beta\right\}$. For each $(A, t) \in P_{n} \times I$ and $\tau \in \operatorname{Sd}^{n} K$ with $\operatorname{dim} \tau=1$, if $t \beta \geq \frac{1}{2} \cdot \operatorname{diam}(\tau \backslash A)$ then $\tau \cap A \neq \varnothing$ and $\hat{\tau} \in \kappa(A, t)$, where $\operatorname{diam} \varnothing=0$. Then we can define a map $\eta: P_{n} \times I \rightarrow P_{n+1}$ as follows:

$$
\begin{aligned}
\eta(A, t)=\kappa(A, t) \cup\left\{x \in\left|\mathrm{St}\left(\hat{\tau}, \mathrm{Sd}^{2 n+1} K\right)^{1}\right| \mid \tau \in \mathrm{Sd}^{2 n} K,\right. \\
\left.\operatorname{dim} \tau=1, \rho^{\prime}(x, \hat{\tau}) \leq t \beta-\frac{1}{2} \cdot \operatorname{diam}(\tau \backslash A)\right\} .
\end{aligned}
$$

And we define $\zeta: P_{n} \times I \rightarrow P_{n+1}$ as follows:

$$
\begin{aligned}
\zeta(A, t)=A \cup\left\{x \in\left|\mathrm{St}\left(v \hat{\tau}, \mathrm{Sd}^{2 n+2} K\right)^{1}\right| \mid v \lesseqgtr \tau \in\left(\mathbf{S d}^{2 n} K\right)^{1},\right. \\
\left.\tau \backslash A \neq \varnothing, 0<\rho^{\prime}(x, v) \leq t \cdot \delta(A, \tau, v)\right\},
\end{aligned}
$$

where

$$
\delta(A, \tau, v)=\frac{\rho^{\prime}(v, \tau \backslash A) \cdot \operatorname{diam}(\tau \backslash A) \cdot \beta}{(\operatorname{diam} \tau)^{2}}<\beta .
$$

It is straightforward to see that $\zeta$ is continuous. The desired embedding $\xi$ : $P_{n} \times I \rightarrow P_{n+1}$ can be defined by $\xi(A, t)=\eta(A, t) \cup \zeta(A, t)$. In fact, each $A \in P_{n}$ contains some 1-simplex $\tau \in \operatorname{Sd}^{n} K$ and then

$$
\rho^{\prime}\left(\hat{\tau},\left|\mathrm{St}\left(\hat{\tau}, \mathrm{Sd}^{2 n+1} K\right)^{1}\right| \backslash \xi(A, t)\right)=t \beta .
$$


Hence $t \neq t^{\prime}$ implies $\xi(A, t) \neq \xi\left(A^{\prime}, t^{\prime}\right)$ for any $A, A^{\prime} \in P_{n}$. If $A \neq A^{\prime} \in P_{n}$ then $\dot{\tau} \cap A \neq i \cap A^{\prime}$ for some 1-simplex $\tau \in \operatorname{Sd}^{n} K$. In case $\operatorname{diam}(\tau \backslash A) \neq$ $\operatorname{diam}\left(\tau \backslash A^{\prime}\right), \eta(A, t) \neq \eta\left(A^{\prime}, t\right)$. In case $\operatorname{diam}(\tau \backslash A)=\operatorname{diam}\left(\tau \backslash A^{\prime}\right) \quad(>0)$, $\delta(A, \tau, v) \neq \delta\left(A^{\prime}, \tau, v\right)$ for at least one vertex $v$ of $\tau$, so $\zeta(A, t) \neq \zeta\left(A^{\prime}, t\right)$. Thus $A \neq A^{\prime}$ implies $\xi(A, t) \neq \xi\left(A^{\prime}, t\right)$ for each $t \in[0,1]$. Therefore $\xi$ is an embedding. By definition, $\xi(A, 0)=A \subset Y_{n}$ for each $A \in P_{n}$.

3.4. Lemma. $\bigcup_{n \in \mathbf{N}} P_{n}$ is dense in $C(X)$.

Proof. For each $A \in C(X)$ and $\varepsilon>0$, choose $n \in \mathbf{N}$ so that $\operatorname{mesh}\left(\operatorname{Sd}^{2 n} K\right)<\varepsilon$ and set $B=Y_{n} \cap \bigcup\left\{\tau \in \operatorname{Sd}^{2 n} K \mid A \cap \tau \neq \varnothing\right\} \in P_{n}$. Then $\rho_{H}(A, B)<\varepsilon$. Therefore $\bigcup_{n \in \mathrm{N}} P_{n}$ is dense in $C(X)$.

\subsection{Lemma. $\bigcup_{n \in \mathrm{N}} P_{n}$ is an fd-cap set for $C(X)$.}

Proof. By Lemma 3.2, each $P_{n}$ is an fd compact $Z$-set in $C(X)$. Let $A \supset B$ be fd compacta, $f: A \rightarrow C(X)$ a map such that $f \mid B: B \rightarrow P_{n}$ is an embedding into some $P_{n}$, and let $\varepsilon>0$. Note that in Lemma $3.1 \mathscr{B} \subset P_{m}$ if $\mathscr{A} \subset P_{m}$. Similar to [GH, Lemma 2], by using Lemmas 3.4 and 3.1, we can construct a map $g: A \rightarrow P_{m}$ of $A$ into some $P_{m} \supset P_{n}$ such that $\rho_{H}(g, f)<\varepsilon / 2$ and $g|B=f| B$. As in the proof of [CN, Lemma 4.6], by using Lemma 3.3 we can replace $g$ by an embedding $h: A \rightarrow P_{l}$ of $A$ into some $P_{l} \supset P_{m}$ such that $h|B=g| B=f \mid B$ and $\rho_{H}(h, g)<\varepsilon / 2$ so $\rho_{H}(h, f)<\varepsilon$. Therefore $\bigcup_{n \in \mathbf{N}} P_{n}$ is an fd-cap set for $C(X)$.

Now we complete the proof of the second part of the Main Theorem. Since $\bigcup_{n \in \mathrm{N}} P_{n} \subset C^{\mathrm{Pol}}(X)$, the result follows from Lemmas 3.5, 2.1, and 3.2, and [Ch, Lemma 4.2].

\section{STAR HYPERSPaCES}

Recall that $K$ is a finite connected simplicial complex with $|K|=X$. In $\left[\mathrm{CS}_{2}\right]$, Curtis and Schori introduced the star hyperspace $2_{\text {sst }}^{K}$ of $K$ by restricting the size of compacta in $2^{X}$ as follows. For each $v \in K^{0}$, let

$$
\operatorname{sst}(v)=\bigcup\left\{\tau \in \operatorname{Sd}^{2} K|| \operatorname{St}(v, \operatorname{Sd} K) \mid \cap \tau \neq \varnothing\right\} .
$$

We define the following star hyperspaces.

$$
\begin{aligned}
& 2_{\text {sst }}^{K}=\left\{A \in 2^{X} \mid A \subset \operatorname{sst}(v) \text { for some } v \in K^{0}\right\} ; \\
& C_{\mathrm{sst}}(K)=C(X) \cap 2_{\mathrm{sst}}^{K} ; \quad C_{\omega s \mathrm{st}}(K)=C_{\omega}(X) \cap 2_{\mathrm{sst}}^{K} ; \\
& \operatorname{Pol}_{\mathrm{sst}}(K)=\operatorname{Pol}(X) \cap 2_{\mathrm{sst}}^{K} ; \quad C_{\mathrm{sst}}^{\mathrm{Psl}}(K)=C^{\mathrm{Pol}}(X) \cap 2_{\mathrm{sst}}^{K} ; \\
& \mathscr{F}_{\mathrm{sst}}(K)=\mathscr{F}(X) \cap 2_{\mathrm{sst}}^{K} .
\end{aligned}
$$

In $\left[\mathrm{CS}_{2}\right]$, Curtis and Schori proved that $2_{\text {sst }}^{K} \cong X \times Q$ and that $C_{\text {sst }}(K) \cong X \times Q$ if $X$ has no free arcs. This is generalized as follows. 
4.1. Theorem. For any finite connected simplicial complex $K$,

$$
\begin{gathered}
\left(2_{\text {sst }}^{K}, \mathscr{F}_{\text {sst }}(K)\right) \cong(|K| \times Q,|K| \times \sigma), \\
\left(2_{\text {sst }}^{K}, \mathrm{Pol}_{\text {sst }}(K)\right) \cong(|K| \times Q,|K| \times \sigma) .
\end{gathered}
$$

In case $K$ has no principal 1-simplex,

$$
\begin{gathered}
\left(2_{\text {sst }}^{K}, C_{\omega \text { sst }}(K)\right) \cong(|K| \times Q,|K| \times \Sigma), \\
\left(C_{\text {sst }}(K), C_{\text {sst }}^{\text {Pol }}(K)\right) \cong(|K| \times Q,|K| \times \sigma) .
\end{gathered}
$$

This theorem is valid for a locally finite simplicial complex $K$ without isolated vertices. To prove the theorem, we need the relative version of $\left[\mathrm{CS}_{2}\right.$, Theorem 3.5]. A locally finite closed cover $\mathscr{D}=\left\{Q_{i}\right\}$ of a metric space $M$ is called a $Q$-decomposition if (i) each $Q_{i}$ is homeomorphic to $Q$; (ii) $\mathscr{D}$ contains all nonempty intersections of its members; and (iii) if $Q_{j}$ is a proper subset of $Q_{k}$, then $Q_{j}$ is a $Z$-set in $Q_{k}$. We say that $Q$-decompositions $\left\{Q_{i}\right\}$ and $\left\{Q_{i}^{\prime}\right\}$ of spaces $M$ and $M^{\prime}$ are isomorphic if they are order isomorphic with respect to the partial orderings given by set inclusion. It is straightforward to see the following version of $\left[\mathrm{CS}_{2}\right.$, Theorem 3.5].

4.2. Theorem. Let $\left\{Q_{i}\right\}$ and $\left\{Q_{i}^{\prime}\right\}$ be isomorphic Q-decompositions of spaces $M$ and $M^{\prime}, N \subset M$ and $N^{\prime} \subset M^{\prime}$ such that each $Q_{i} \cap N$ and $Q_{i}^{\prime} \cap N^{\prime}$ are fd-cap sets (or cap sets) for $Q_{i}$ and $Q_{i}^{\prime}$, respectively. Then there exists a homeomorphism of $M$ onto $M^{\prime}$ taking each element of $\left\{Q_{i}\right\}$ onto the corresponding element of $\left\{Q_{i}^{\prime}\right\}$ and $N$ onto $N^{\prime}$.

Proof of Theorem 4.1. For $A_{1}, \ldots, A_{m} \in 2^{X}$, let

$$
\begin{aligned}
2^{X}\left(A_{1}, \ldots, A_{m}\right) & =\left\{A \in 2^{X} \mid A \cap A_{i} \neq \varnothing \text { for each } i\right\}, \\
C\left(X ; A_{1}, \ldots, A_{m}\right) & =C(X) \cap 2^{X}\left(A_{1}, \ldots, A_{m}\right) .
\end{aligned}
$$

First, by [CN, Corollary 5.2], $\mathscr{F}\left(X ; A_{1}, \ldots, A_{m}\right)=\mathscr{F}(X) \cap 2^{X}\left(A_{1}, \ldots, A_{m}\right)$ is an fd-cap set for $2^{X}\left(A_{1}, \ldots, A_{m}\right)$. As in $\S 2$, we can see that

$$
C_{\omega}\left(X ; A_{1}, \ldots, A_{m}\right)=C_{\omega}(X) \cap 2^{X}\left(A_{1}, \ldots, A_{m}\right)
$$

is a $Z_{\sigma}$-set in $2^{X}\left(A_{1}, \ldots, A_{m}\right)$, so

$$
\operatorname{Pol}\left(X ; A_{1}, \ldots, A_{m}\right)=\operatorname{Pol}(X) \cap 2^{X}\left(A_{1}, \ldots, A_{m}\right)
$$

is also an fd-cap set for $2^{X}\left(A_{1}, \ldots, A_{m}\right)$. Similar to $\left[\mathrm{CS}_{2}\right.$, Theorem 4.3], the first half of the theorem follows from Theorem 4.2.

In the case where $X$ has no free $\operatorname{arcs}, C_{\omega}\left(X ; A_{1}, \ldots, A_{m}\right)$ is a cap set for $2^{X}\left(A_{1}, \ldots, A_{m}\right)$ by the result of $\left[\mathrm{Mi}_{2}\right]$. By choosing $B_{i}$ in the proof of Lemma 3.2 so that $B_{i} \cap A_{j}=\varnothing$ or $B_{i} \varsubsetneqq A_{j}$ for each $j=1, \ldots, m$, we can prove that

$$
C^{\mathrm{Pol}}\left(X ; A_{1}, \ldots, A_{m}\right)=C^{\mathrm{Pol}}(X) \cap 2^{X}\left(A_{1}, \ldots, A_{m}\right)
$$


is a $Z_{\sigma}$-set for $C\left(X ; A_{1}, \ldots, A_{m}\right)$. Now we assume that each $A_{i}$ is a polyhedron triangulated by a subcomplex of $\operatorname{Sd}^{2} K$. Then each $A_{i}$ meets each $Y_{n}$ defined in $\S 3$. For each $n \in \mathbf{N}$, let $P_{n}\left(A_{1}, \ldots, A_{m}\right)=P_{n} \cap 2^{X}\left(A_{1}, \ldots, A_{m}\right)$, where $P_{n}$ is defined in $\S 3$. Then we can prove, in a way similar to Lemma 3.5, that $\bigcup_{n \in \mathrm{N}} P_{n}\left(A_{1}, \ldots, A_{m}\right)$ is an fd-cap set for $C\left(X ; A_{1}, \ldots, A_{m}\right)$. Since $\bigcup_{n \in \mathbf{N}} P_{n}\left(A_{1}, \ldots, A_{m}\right) \subset C^{\mathrm{Pol}}\left(X ; A_{1}, \ldots, A_{m}\right), C^{\mathrm{Pol}}\left(X ; A_{1}, \ldots, A_{m}\right)$ is an fd-cap set for $C\left(X ; A_{1}, \ldots, A_{m}\right)$. Therefore the second half also follows from Theorem 4.2.

\section{REMARKS}

In the case where $X$ is a finite graph, we have $\operatorname{Pol}(X)=C_{\omega}(X)$ and $C^{\mathrm{Pol}}(X)=C(X)$. The first assertion of the theorem has been shown in this case by Michael $\left[\mathrm{Mi}_{1}\right]$ (cf. $\left.\left[\mathrm{Mi}_{2}\right]\right)$, and the assumption of the second statement is essential.

The second part of the Main Theorem can be generalized as follows.

5.1. Theorem. If $X$ is a compact connected polyhedron with no free arc, then for each $n \in \mathbf{N},\left(C_{n}(X), C_{n}(X) \cap \operatorname{Pol}(X)\right) \cong(Q, \sigma)$.

Proof. First note that in Lemma 3.1, $\mathscr{B} \subset C_{n}(X) \cap \operatorname{Pol}(X)$ if $\mathscr{A} \subset C_{n}(X) \cap$ $\operatorname{Pol}(X)$. In the proof of Lemma 3.2, the map $\varphi$ can be defined on $2^{X}$. In this case, we have $\varphi\left(C_{n}(X)\right) \subset C_{n}(X)$ for each $n \in \mathbf{N}$. Hence it follows that $C_{n}(X) \cap \operatorname{Pol}(X)$ is also a $Z_{\sigma}$-set in $C_{n}(X)$ for each $n \in \mathbf{N}$ if $X$ has no free arc. As is easily seen, $C_{n}(X) \cap \operatorname{Pol}(X)$ is dense in $C_{n}(X)$. For each $m, n \in \mathbf{N}$, let $Y_{m}=\left|\left(\operatorname{Sd}^{m} K\right)^{1}\right|$ and $P_{n, m}=\left\{A \in C_{n}\left(Y_{m}\right) \mid A\right.$ contains some $\left.\tau \in\left(\operatorname{Sd}^{m} K\right)^{1}\right\}$. As in $\S 3$, we can prove that $\bigcup_{m \in \mathrm{N}} P_{n, m}$ is an fd-cap set for $C_{n}(X)$. For each $n \in \mathbf{N}, C_{n}(X) \cong Q$ and $\bigcup_{m \in \mathbf{N}} P_{n, m} \subset C_{n}(X) \cap \operatorname{Pol}(X)$. (E.g., $C_{n}(X) \cong Q$ follows from [Cu, Corollary 5.1] since it is as easy to show as in Lemma 3.2 that $\{X\}$ is a $Z$-set in $C_{n}(X)$.) Thus we have the result similar to the second part of the Main Theorem.

From Theorem 5.1, it is natural to conjecture as follows.

5.2. Conjecture. For a compact connected polyhedron $X$ with no free arc, $\left(2^{X}, C_{\omega}(X), \operatorname{Pol}(X)\right) \cong(Q, \Sigma, \sigma)$.

Applying the characterization of the triple $(Q, \Sigma, \sigma)$ in [SW], this conjecture is reduced to show that each $C_{n}(X)$ is a $Z$-set in some $C_{m}(X)$ (cf. [Sa]). And related to the result of $\S 4$, it is also natural to conjecture as follows.

5.3. Conjecture. For a (locally) finite simplicial complex $K$ with no principal 1 -simplexes,

$$
\left(2_{\text {sst }}^{K}, C_{\omega \text { sst }}(K), \mathrm{Pol}_{\text {sst }}(K)\right) \cong(|K| \times Q,|K| \times \Sigma,|K| \times \sigma) .
$$

One may also conjecture that $\left(2^{X}, C_{\omega}(X), \mathscr{F}(X)\right) \cong(Q, \Sigma, \sigma)$ since $\mathscr{F}(X)$ $\subset C_{\omega}(X) \subset 2^{X},\left(2^{X}, C_{\omega}(X)\right) \cong(Q, \Sigma)$, and $\left(2^{X}, \mathscr{F}(X)\right) \cong(Q, \sigma)$. However 
this conjecture is false. In fact, $C_{\omega}(X) \backslash \mathscr{F}(X)=\bigcup_{n \in \mathbf{N}} C_{n}(X) \backslash \mathscr{F}(X)$ and each $C_{n}(X) \backslash \mathscr{F}(X)$ is $\sigma$-compact because $\mathscr{F}(X) \cap C_{n}(X)=\mathscr{F}_{n}(X)$ is compact, so it is closed in $C_{n}(X)$. Hence $C_{\omega}(X) \backslash \mathscr{F}(X)$ is also $\sigma$-compact. On the other hand, $\Sigma \backslash \sigma$ is not $\sigma$-compact because $\Sigma \backslash \sigma \cong \Sigma \times s$ by [SW, Theorem 3.1]. This observation is due to Jan van Mill. The author would like to thank Jan van Mill for his observation.

\section{REFERENCES}

[An] R. D. Anderson, On sigma-compact subsets of infinite-dimensional spaces, unpublished.

[Ch] T. A. Chapman, Dense sigma-compact subsets of infinite-dimensional manifolds, Trans. Amer. Math. Soc. 154 (1971), 399-426.

[Cu] D. W. Curtis, Growth hyperspaces of Peano continua, Trans. Amer. Math. Soc. 238 (1978), 271-283.

[CN] D. Curtis and Nguyen To Nhu, Hyperspaces of finite subsets which are homeomorphic to $\aleph_{0}$-dimensional linear metric spaces, Topology Appl. 19 (1985), 251-260.

[CM] D. Curtis and M. Michael, Boundary sets for growth hyperspaces, Topology Appl. 25 (1987), 269-283.

[CS $\left.{ }_{1}\right]$ D. W. Curtis and R. M. Schori, Hyperspaces of Peano continua are Hilbert cubes, Fund. Math. 101 (1978), 19-38.

$\left[\mathrm{CS}_{2}\right]-$, Hyperspaces which characterize simple homotopy type, Gen. Topology Appl. 6 (1976), 153-165.

[GH] R. Geoghegan and W. E. Haver, On the space of piecewise linear homeomorphisms of a manifold, Proc. Amer. Math. Soc. 55 (1976), 145-151.

[Ha] W. E. Haver, Locally contractible spaces that are absolute neighborhood retracts, Proc. Amer. Math. Soc. 40 (1973), 280-284.

[Ke] J. L. Kelley, Hyperspaces of a continuum, Trans. Amer. Math. Soc. 52 (1942), 22-36.

[Me] K. Menger, Untersuchungen über allgemeine Metrik, Math. Ann. 100 (1928), 75-163.

[Mi $]$ M. Michael, Sigma-compact subsets of hyperspaces, Doctoral Dissertation, Louisiana State Univ., 1979.

$\left[\mathrm{Mi}_{2}\right] \quad$ Some hyperspaces homeomorphic to separable Hilbert space, General Topology and Modern Analysis (L. F. McAuley and M. M. Rao, eds.), Academic Press, New York, 1981, pp. 291-294.

[Na] S. B. Nadler, Jr., A characterization of locally connected continua by hyperspace retractions, Proc. Amer. Math. Soc. 67 (1977), 167-176.

[RS] C. P. Rourke and B. J. Sanderson, Introduction to piecewise-linear topology, EMG 69, Springer-Verlag, Berlin, 1972.

[Sa] K. Sakai, A function space triple of a compact polyhedron into an open set in Euclidean space, Proc. Amer. Math. Soc. (to appear).

[SW] K. Sakai and R. Y. Wong, On finite-dimensional manifold triples, Trans. Amer. Math. Soc. 318 (1990), 545-555.

[To] H. Torunczyk, On CE-images of the Hilbert cube and characterization of Q-manifolds, Fund. Math. 106 (1980), 31-40.

Institute of Mathematics, University of Tsukuba, Tsukuba city, 305 Japan 